\title{
Critical Care Nurses' Experiences With Death and Dying: A South African Perspective
}

\author{
Vasanthrie Naidoo ${ }^{1} \&$ Maureen Nokuthula Sibiya ${ }^{2}$ \\ ${ }^{1}$ Faculty of Health Sciences-Nursing, Durban University of Technology, Durban, South Africa \\ ${ }^{2}$ Faculty of Health Sciences, Durban University of Technology, Durban, South Africa \\ Correspondence: Vasanthrie Naidoo, Faculty of Health Sciences-Nursing, Durban University of Technology, \\ Durban, South Africa. Tel: 27-31-373-2748. Email: vasanthrien@dut.ac.za
}

Received: May 7, 2019 Accepted: June 15, 2019 Online Published: July 10, 2019

doi:10.5539/gjhs.v11n9p69

URL: https://doi.org/10.5539/gjhs.v11n9p69

\begin{abstract}
The aim of this study was to explore experiences of South African critical care nurses regarding grief, death and dying in a critical care environment. Data was collected using semi-structured interviews and was analyzed using Giorgi's thematic data analysis method. Available literature suggests that critical care nurses have varied experiences in relation to their experiences in relation to end-of -life patient care. However, few studies have examined the involvement of South African intensive care nurses' in caring for the dying patient, their grief, their reactions to death in the workplace and the extent to which their nursing practice is based on shared beliefs, experiences and attitudes. Findings from this study revealed many predisposing factors and circumstantial occurrences shaping both, the nature of care of the dying and subsequent grief that, affected the nurse. Repeated exposure to grief, leads to occupational stress and burn out, causing emotional disengagement from caring for the dying, which ultimately affect the quality of care rendered for both the dying patient and their family. Issues, such as communication, multicultural diversity, education and coping mechanisms are essential in nursing education and practice and nurses caring for the critically ill or dying patient, need to have support networks and strategies put in place, not only to assist in providing care, but also for their own emotional support and well-being.
\end{abstract}

Keywords: critical care, intensive care, grief, death, dying

\section{Introduction and Background to the Study}

Seriously, ill patients admitted to critical care units or the intensive care units (ICUs), take advantage of advanced technology and intensive nursing care with a primary emphasis on recovery and survival. An admission to ICU creates a crisis to both patients and families. As a result, in addition to the initial crisis leading to admission to critical care, health care professionals and the families have to deal with the management and negotiation of death, which is regarded as highly stressful, and emotionally distressing (Efstathiou \& Clifford, 2011). The risk of sudden imminent death does not allow for 'preparation for death' and places enormous demands on the nurses who are expected to provide high-quality care for both the dying and the bereaved (de Beer, Brysiewicz, \& Bhengu, 2011). Available literature suggests that critical care nurses have varied experiences in relation to their experiences in relation to grief and end-of-life patient care, stating that nurses tend to provide continuous health care to ill patients until they die, while doctors consult patients occasionally (Kgosana, Mamogobo, Mothiba, \& Okafor, 2019). The same study reported that, even though ppatients required physical, psychological and social care from the nurses, the impact of caring for critically ill patients and their families, might prove to be overwhelming if support systems, particularly in the work environment, are not in place and the nurses are not taken care of.

Current information informs nursing practice and education that critical care nurses play a crucial role in providing care to critically ill and dying patients and their families and grief, death and dying in an ICU can have a negative impact on the psychosocial well-being of the nurse.However,few studies have examined the involvement of South African critical care nurses' in caring for the dying patient, their grief, reactions to death in a critical care environment and the extent to which their nursing practice is based on shared beliefs, experiences and attitudes. The authors of this paper envisage the findings to provide insight into cultural and personal values held by South African ICU nurses on death and dying and its impact on end-of-life care, validating the need to have ICU support networks and strategies put in place, for nurses' emotional support and well-being. It also addresses the need for death education to be emphasized in the South African nursing undergraduate nursing curriculum and continue to 
be integrated to post-graduate or post basic nurse training such as ICU nursing. The aim of this study was, therefore to explore the South African critical care nurse's experiences of death and dying in the ICU.

\subsection{The Critical Care/Intensive Care Environment}

According to Hov, Hedelin and Athlin (2005), in many ways, 'intensive care' represents, in a symbolic and real sense, the modern preoccupation with the mastery of disease, the eradication of 'untimely death' and the prolongation of life. The goal of these ICUs or critical care units has long been viewed as assisting the individual to survive a life-threatening physiological process. Integral to that survival is the critical care nurse who, with the assistance of advanced assessment modalities, multiple pharmacological agents, and sophisticated technology, directs the care of the patient and the family (Carlson, 2009). The environment of an intensive-care unit has been reco0gnized as a very stressful, high-tech, fast paced and emotionally charged atmosphere. According to Prompahakul, Nilmanat, and Kongsuwan (2011), nursing in an ICU can influence a critical care nurses' experiences with a dying patient or the end-of -life care. According to Urden, Stacy and Lough (2010), even though critical care nurses serve in specialty roles that require their clinical teaching, leadership, research and consultative abilities, keeping the 'care' in nursing is one of the biggest challenges of critical care nursing. This challenge is heightened with grief, death and dying because not only must the critical nurse be able to deliver high quality care skillfully, using all appropriate technologies, she must also be able to apply psychosocial and other holistic approaches when planning and delivering care (Urden, Stacy, \& Lough, 2004).

\subsection{Global Perspectives of Coping With ICU Grief}

Patients enter the acute care setting in physiological crisis and treatment can be sometimes aggressive in order for it to be lifesaving, but it is estimated that, as many as one in five patients dies in a critical care unit (Carlson, 2009). Prompahakul, Nilmanat, and Kongsuwan (2011), maintain that caring for patients at the end of life becomes an indicator of the quality of care in a hospital. For most nurses the loss of a patient can create intense feelings of grief, and for others the reminder of their own personal tragedies creates a very trying and stressful situation for the critical care nurse. According to Michell (2010), nurses suffering from such stress may lose their capacity to perform well in their job and this can result in job dissatisfaction and burnout due to repetitive exposure to resuscitative measures. Burnout is a term used to describe a prolonged response to chronic psychosocial stressors in one's work and is considered, a multidimensional syndrome that occurs primarily in professions where the practitioners are working closely with people in intense and stressful interactions (Jacobsson, Backteman-Erlanson, Padyab, Egan Sjolander and Brulin, 2016). According to Liebert (2011), acute grief following the death of a loved one or patient in ICU is often intensely painful but can diminishes over time as the loss becomes integrated into ongoing life and the general nursing routine. The study also revealed that, adjusting to a loved one's death required both parties; the nurse as a caregiver and the loved ones, to acknowledge death as a reality. Langley and Schmollgruber (2006) noted that at the end-of-life, the patient, the family, doctors and nurses are the primary persons involved in the actual ethical decision-making process and being the human face of care, decision-making is frequently a frustrating process with conflicting morals and values coming to the fore. In a study done by Wingate and Wiegand (2008), it was reported that most deaths in a hospital, occur in a critical care unit and that American views on death and dying are very much influenced by Western medicine. The American Association of Critical Care Nurses has maintained that critical illnesses should never become an ordinary experience for nurses and knowledge and care go hand in hand. Therefore, the specialty of critical care requires high levels of preparation and in-depth knowledge to enable the critical care nurse to cope with death and dying (Alspach, 2006). Goodridge et al., (2009), agrees that the implementation of thoughtful and relevant strategies to conceptualize and measure quality of end-of-life care in the ICU will prove of significant benefit to all health professionals involved in critical care. According to Niederriter (2009), surveys done by the Canadian Nurses Association (CNA) revealed that, nurses are probably one of the most important role-players in an ICU because they assist and provide direction to the dying patient. They form part of a complex process that is a physically, psychologically, emotionally and a spiritually intimate one, which allows them to develop a therapeutic relationship with the dying patient. Kongsuwan, Chaipetch and Matchim (2012), explored the process of promoting a peaceful death for dying patients in ICUs in Thailand and found that ICU nurses used their experiences of caring for many deaths and the knowledge of the dying process to anticipate impending death of their patients. A Swedish study done by Fridh, Forsberg and Bergbom (2007), reported that the critical care nurse's knowledge of the Swedish culture of death and dying and family presence in an ICU at the time of death was seen as effective end-of-life care. The findings of an Australian study reported that critical care nurses can sometimes feel powerless to change events that affect dying patients and intensive care practitioners are often deeply concerned with issues of human dignity, and with death and dying issues in ICUs (Kirchhoff \& Beckstrand, 2005). Latour, Fulbrook and Albarran (2009), found that the experiences and attitudes of critical care nurses demonstrated a shared outlook with differences in some areas, 
which may be a reflection of culture, religious view and educational preparation of nurses or approaches to service delivery. According to Vailee, Negararandeh and Dehghan Nayeri (2012), nurse's experiences with patients and end-of-life was accompanied by emotional burden and a conflict in values and beliefs and concluded that there is a need to increase the current knowledge related to providing care to end-of-life patients at ICUs. The same authors emphasized the need for providing the nurses with psychological support and education that will allow nurses to manage the conditions of the patients and their families while engaging in decision making about the dying patient.

\section{Methods}

\subsection{Study Design and Its Philosophical Underpinning}

In order to explore the experiences of the participants with death and dying, a qualitative, descriptive phenomenological approach was used to guide the study. Phenomenology differs from other approaches due to its emphasis on the participants' experienced meaning rather than just on a description of their observed behaviors or actions. This supports the subjective aspect of human activity by focusing on the meaning rather than the measurement of the social phenomena. The descriptive phenomenological research method used in this study, therefore, attempted to uncover the underlying essences and meanings of experience to arrive at a deeper understanding. Polit and Hungler (2004), define this concept by stating that, it is basic approach to thinking about what the life experiences of people are really like.

\subsection{Study Site}

This study was conducted in the ICU of a large 543 bedded provincial hospital in the Chatsworth area in the eThekwini District in the province of KwaZulu-Natal (KZN) (KZN Department of Health, 2011). Chatsworth is a suburb located in the southern eThekwini region with a population of approximately 192000 thousand citizens. The selected hospital in this study is a health care service provider to areas in and around Chatsworth and serves a very diverse and multicultural population as well as mixed income groups namely, the upper, middle and lower class groups. The ICU is a general ICU and admits patients from various disciplines such as medical, surgical and orthopedic emergencies. This unit very often accepts patient overflows from 'sister' hospitals' critical care units when there is a shortage of patient beds at those facilities.

\subsection{Population and Sampling}

A criterion sampling of all consenting professional nurses employed in the ICU of the participating hospital took part in the study. The hospital has a total population of 15 staff members in the ICU. Of these 15, ten are professional nurses of whom only six are ICU trained. Other categories included four enrolled nurses and one enrolled nursing assistant. There are four shifts or teams that run concurrently at any given time. The two-day duty nursing shifts that work on alternate days to each other have only two ICU trained or qualified professional nurses on each shift or team. The two-night duty nursing teams that also work on opposite shifts are only staffed with one ICU trained or qualified professional nurse on each team. Participants for the study had to be critical care trained/experienced professional nurses working in the ICU for a minimum of one year and had to be a permanent employee of the participating hospital.

\subsection{Data Collection}

Qualitative data was collected by means of in-depth interviews after obtaining informed written consent from participants. For the purposes of this research, one face-to-face interview was conducted with the critical care nurse and one broad question was asked: 'what are your experiences regarding death and dying of your patients in ICU?' Participants were asked about their experiences of providing care for the critically ill and the impact it had on their work performance and personal lives. Subsequent questions were framed around their responses and participants were invited to contribute other points that were not raised by the interviewer. Data was collected until the researcher was satisfied that the data collection process they had reached the point of saturation and no new concepts or ideas were being generated.

\subsection{Data Analysis}

Data were analyzed using Giorgi's qualitative data analysis as outlined by Creswell (2013). After analysis of the in-depth interviews with the critical care nurses, a thematic framework was used to categorize findings into themes and sub-themes according to the objectives of the study. The researcher was able to get both objective and subjective responses from the participants that provided both their professional and personal reflections and attitudes on death and dying. There researcher endeavored to keep the richness of the experience that each participant had with the patients that they cared for, whilst exploring the descriptive meanings of such experiences, (Polit \& Beck, 2008). 


\subsection{Rigour and Trustworthiness}

Trustworthiness in qualitative research means methodological soundness and adequacy. Trustworthiness can be assessed through the attributes of dependability, credibility, transferability and confirmability within the data (Polit $\&$ Beck, 2008). In this study, the element of rigour was achieved through member checking, peer debriefing, triangulation, an audit trial, providing thick descriptions with which to illustrate the emerging concepts and reflexivity (Polit \& Beck, 2008). Having sufficient time for data collection and analysis, building a rapport with the participants and involving them in participant verification, peer review and validation of emergent themes were important features of the work.

\subsection{Ethical Considerations}

Ethical clearance was obtained from the Durban University Institutional Research Ethics Committee (IREC), whilst permission to collect data in the health facility was obtained from the Department of Health, KZN Province. The researcher obtained informed consent, in writing, from all participants before conducting the interview sessions. One of the consequences of participating in a study of this nature was the sensitivity of the concepts discussed under the topic of inquiry. Interviews and discussion with participants evoked a certain amount of distress causing mental anguish and deep-seated anger from the pent up emotions that participants appeared to harbor. The personal losses of loved ones seemed to trigger emotional outbursts and episodes of crying whilst being interviewed. This resulted in debriefing sessions being held after the interviews by the interviewer.

\section{Results}

Qualitative content analysis yielded five main themes and its related sub-themes (Table 1).

Table 1. Critical care nurses' experiences with death and dying

\begin{tabular}{|c|c|}
\hline Main Themes & Sub-themes \\
\hline $\begin{array}{l}\text { Critical care nurses' thoughts about } \\
\text { caring for a dying patient. }\end{array}$ & $\begin{array}{l}\text { - } \quad \text { Age of patient and its role in death acceptance. } \\
\text { - } \quad \text { Age of nurse and its role in determining maturity and understanding. }\end{array}$ \\
\hline $\begin{array}{l}\text { Critical care nurses' feelings about } \\
\text { caring for a dying patient. }\end{array}$ & $\begin{array}{l}\text { - } \quad \text { Feelings of grief when coping with the dying or dead patient. } \\
\text { - } \quad \text { Feelings of knowing when to prepare for inevitable death of a patient. }\end{array}$ \\
\hline $\begin{array}{l}\text { Critical care nurses' communication } \\
\text { with dying patients. }\end{array}$ & $\begin{array}{l}\text { - Communication with dying patient. } \\
\text { - Communication with loved ones of dying patient. } \\
\text { - Communication of cultural awareness. } \\
\text { - } \quad \text { Non-verbal communication. }\end{array}$ \\
\hline $\begin{array}{l}\text { Past experiences with death and dying } \\
\text { and the impact it had on the critical care } \\
\text { nurse. }\end{array}$ & $\begin{array}{l}\text { - The personal impact of death and dying on the critical care nurse. } \\
\text { Exposure of critical care nurse to death and dying and the role it plays in } \\
\text { coping. }\end{array}$ \\
\hline $\begin{array}{l}\text { Support systems that enable the critical } \\
\text { care nurse to cope with the trauma of } \\
\text { death and dying. }\end{array}$ & $\begin{array}{l}\text { - } \quad \text { Lack of workplace structures. } \\
\text { - } \quad \text { Use of spirituality as support and guidance. } \\
\text { - } \quad \text { Lack of nurse education on death and dying issues. }\end{array}$ \\
\hline
\end{tabular}

\subsection{Critical Care Nurses' Thoughts About Caring for a Dying Patient}

It was apparent from the participants' responses that the thoughts on death of an elderly or aged patient often crossed a critical care nurse's mind. The concept of death being inevitable or real seemed to provide some measure of comfort to the nurse caring for the patient. When youngsters or children died, it becomes very sad and unmanageable at times for all staff in an ICU. The fact that it was a young person involved tends to make the whole situation highly emotional. The age of the nurse caring for such a patient seemed to have a bearing on the way the nurse copes with the event. Younger nurses were found to be task orientated, and lacked the empathy, respect and psychosocial aspect of patient care. Older nurses were considered to be the most suitable candidates to deal with death and dying issues in the unit as they have more 'humane attributes and qualities stemming from her life's experiences as well as having sound technical and theoretical skills'. This is supported by the following statements. 


\section{"I think that adult patients have seen life" Participant 1}

"Young nurses seem to be totally unaffected by the impact of a dying patient on his or her family". Participant 2

"With the aged, you actually think that they shouldn't suffer for long and when you look at them, you realize that death and dying is a process of life." Participant 4

\subsection{Critical Care Nurses' Feelings About Caring for a Dying Patient}

The pain of watching a loved one die or caring for a loved one throughout the dying process can evoke feelings of despair, anger and even denial. Apart from playing the important role of being a patient advocate, the findings of this study revealed that the critical care nurse felt ethically responsible to do his or her best. Feelings of preparedness allowed the nurse to foresee or predict, using their nursing knowledge, the prognosis of a critically ill patient in their care. This helped them prepare themselves and others for what to expect. Participants in this study have illustrated this finding as follows:

"You become so attached to your patients and their relatives that you actually feel quite sad when they die." Participant 2

"I don't feel that death is easy to accept whether it was a long term patient or a short term patient." Participant 1

"But, you know, as long as the family members come, you bond with them. You update them and when a person dies, you feel as though it was your own" Participant 3

\subsection{Critical Care Nurses' Communication With Dying Patients}

The findings of this study also revealed that the nurse who provided verbal and non-verbal communication with her patients, allowed her to become more in tune with the needs of the patient and their desires as they neared death. According to research findings from a study that examined cultural sensitivity amongst nurses, it was found that culture plays a vital part in end-of- life decision-making. Responses from participants in this study, demonstrated that a critical care nurse needed to be aware of cultural attitudes, behaviors and traits of patients to enhance the caring component in critical care nursing. The following statements made by participants confirm this:

"Dying people have the sense of fear so just being around them and holding their hand and saying some soothing words, helps the dying process". Participant 2

"Well, there are no words that can offer comfort but if you just hold that person, give them a tissue to wipe the tears, stand there with them. It a kind of silent communication". Participant 1

\subsection{Past Experiences With Death and Dying and the Impact It Had on the Critical Care Nurse}

Research findings from a study focusing on resuscitative measures in hospital high -tech environments, revealed that repetitive exposure to resuscitative measures, end-of-life care needs, prolonging life by pharmacological and mechanical means results in psychological disorders such as post-traumatic stress disorders. All the participants that were interviewed felt that a critical care nurse's personal experiences with death and dying impacted on the way she communicated or related to her patients and their loved ones. Participants further stated that the knowledge and skills gained from their personal experiences of caring for a dying family member or any other person that they were associated with impacted on the way they communicated verbally and non-verbally with their patients and the family of their patients. The following excerpts from the actual interview responses confirm this.

"Because it hurts me when a patient dies as I lost both my parents" Participant 4

"My brother committed suicide so when other patients die of suicide attempts I am moved". Participant 3

\subsection{Support Systems That Enable the Critical Care Nurse to Cope With the Trauma of Death and Dying}

ICU nurses experience death in a critical care environment as a series of inter-relationships between patients, nurses, doctors and other members of the multidisciplinary team. This enabled them not only to support the dying patient or the patients' family, but also to support each other in the face of grief. However, the collaboration between ICU caregivers and member of the multidisciplinary team can result in timelier decisions regarding life-sustaining treatments. Acute grief following the death of a loved one or a patient in ICU is often intensely painful but diminishes over time as the loss becomes integrated into ongoing life and the general nursing routine. The findings in this study revealed that, despite advances in the healthcare sector, the critical care nurse is not being sufficiently equipped with skills to deal with death and dying in their professional practice, especially if the nurse had little or no exposure dealing with death and dying issues. This was supported by strong viewpoints regarding the active participation of the patient's attending doctor in the end-of -life care of their patients, as well 
as in keeping the patient and relatives fully informed of the patient's progress. Participants felt that the skills that had been acquired by them were learned through their personal and life's experiences. It was also felt that other resources such as social workers, counsellors and designated grieving areas were needs that were still outstanding in the hospital ICU set-up. Interviewees aired their views as follows:

"We should employ specific people to help colleagues and nursing personnel to deal with stressful issues in an ICU”. Participant 4

"Strategies should be implemented to help ICU nurses with coping mechanisms". Participant 2

\section{Discussion}

After the analysis of the data, this study indicated that nurses working with ill patients in an ICU had various thoughts about working with dying patients and their families including the fact that it can be frustrating and emotionally draining for all concerned parties. This suggests that the many difficulties that arise when working with such patients, such as communication difficulties and ethical dilemmas only make the critical care nurses more determined to function in the capacity of a patient advocate and assist the patient with all his or her daily activities of living, despite the somewhat painful and stressful job of an ICU sister (Kirchhoff \& Beckstrand, 2005: 395). This study also revealed the conflicting thoughts that arise from caring for the dying related to the different age groups of the patient and the nurse caring for the patient. These findings were consistent with a study done by Glaser and Strauss (2004: 174) who examined the impact of younger patients' death on nurses. These authors found that when younger patients died, every possible thought that entered a critical care nurse's mind focused on helping this patient onto the road to recovery. With older patients, however it was thought that they had experienced their lives. Even though the findings of this study have shown that any experience with death or caring for the dying was emotionally draining, it was in keeping with the literature review that critical care nurses became frustrated and were uncertain whether their role was to postpone death or extend life. Fear was something all critically ill patients faced. Nursing a critically ill patient was just as daunting and fearful to the nurse, especially when death was inevitable. Many respondents in this study agreed that ICU nurses became angry with the whole situation when a patient died as they felt that all their efforts had been in vain or that "life was just unfair". To some nurses, there was still a feeling of guilt that more should have been done to help the patient. Despite the challenges of proper communication with dying patients, this study revealed that critical care providers wish to avoid the problems associated with communication breakdowns. These health care providers consider sensitive communication a priority and make time to integrate communication into caring for a critically ill patient. The findings of this study was in agreement with literature from Curtis (2001: 363) which stated that discussing end-of-life care and death with patients and their families was an extremely important part of providing a good quality care in the ICU. Nurses often appear to express a sense of helplessness, frustration, uselessness and guilt when working with patients and families in death and dying situations. Although there is really nothing a human being can do to stop the destiny process of another human being, we can help the dying patient and their families in their final hours with our education and compassion. It was also evident that events such as the death of a family member or friend, one's religious upbringing, near death experiences and personal attitudes can determine a patient's thought processes related to grief, death and dying (Press, Thorn, \& Kline, 2001). The issue of critical care nurses being unprepared to take an active role in caring for patients who are dying or are terminally ill was another fact that surfaced during the data collection process of this research. Understanding critical care nurses' experiences of death and dying can help the employer or the healthcare service delivery system to prepare these nurses for such events by using available research to implement training and development.

The psychosocial impact of grief and loss is briefly covered in the ICU nursing curriculum. This was a point that was brought up by almost all the respondents, who felt that nurses needed to be made aware of and to respect the grieving process of the patient and family and therefore more emphasis should be focused on such issues in the nursing curriculum. Death and dying is a phenomenological experience and sometimes experience is the best teacher. Those nurses who were interviewed, reinforced the notion that ICU nursing curriculums still are not providing adequate end-of-life training and local and international studies have shown that comprehensive end-of-life care training is greatly needed. Participants believe that specific skills related to caring for the dying and the dead need to be incorporated in undergraduate ICU nursing curriculums such as the current Diploma in Medical and Surgical Nursing Science-Critical Care course that is run in South Africa. Communication skills are vital in all aspects of critical care nursing practice and are a vital component when dealing with the patient, the family or any other member of the health team. Very often critical care nurses tend to be emotionally affected by their patients' death especially if they have lost loved ones of their own. Death takes an emotional toll on all persons caring for the dying and therefore, critical care nurses need to have support networks in place, not only to 
assist in providing care, but also for their own emotional wellbeing. Irrespective of religion, culture or race, spirituality plays a huge role when caring for the dying and provides a resource for coping with death and dying for participants in this study. A nurse's age and level of maturity, as well as past experiences with dying and death plays a key role in providing care at end-of-life.

\section{Conclusion}

Whilst death is a very personal event for any patient, it rarely happens in isolation. Death, as a phenomenon, encompasses cultural, ethnic, spiritual, social and physical elements and very often when critical patients are dying or die in an ICU environment, the critical care nurse is present. The researcher hopes that findings of this study will not only indicate that nurses can advocate for improving the care given to dying ICU patients by becoming proactive and involved agents of change within their own organizations. Specific measures that nurses use to support patients and their families through the stress of crisis and adaptation to illness, death, or a return to health sometimes go unnoticed and an understanding and appreciation of the intricate relationships among mind, body, spirit, and the healing process enables the critical care nurse to provide emotional support to the patient and family. It is the caring and emotional support given by the nurse that is often remembered, valued and assists in promotion of quality end of life care in an ICU. The findings of this study could be used to suggest implications for nursing practice, education, policy development and research.

This study set out to explore death and dying experiences of nurses in South African critical care units and to explore the nurses' understanding of their caretaking experiences. Data was gathered using a qualitative exploratory study. The goal was to develop strategies to enable the South African nurses to cope more effectively with the emotional impact of this care both personally and in their professional practice. Critical care nurses relate in different ways to the phenomena of death and dying within their nursing profession and their scope of practice. Therefore, insight into cultural and personal values held by these nurses on death and dying provided a valuable and unique understanding of contemporary practices. This study focused on a fundamental phenomenon (death and dying) in South African critical care nursing. Using a phenomenological research methodology, the researcher was unable to anticipate how the study was going to evolve. Much of the research design appeared was formulated during the data collection and analysis process. Using this approach, helped the researcher to examine the human experience based on the descriptions provided by the participants themselves, which further allowed the meanings these descriptions to add credibility to the study. Participants also stated that having a trained trauma counsellor on site to deal with debriefing and psychological rehabilitation of patients, relative and staff would be a huge benefit to the institution, considering the high volume of critically ill patients the institution admits.

The researcher, being a psychiatric trained nurse, was able to offer assistance in the participant debriefing sessions and found that the verbalization from participants during debriefing sessions assisted them in bringing about a cathartic effect to coping with death and dying not only in the workplace but in everyday life. It was further discovered in the debriefing sessions, that nursing can be an emotional experience and it becomes important for critical care nurses to identify ways to cope with their feelings while working with dying patients. Cognisant of the very demanding nature of the nursing profession, participants were adamant that there was a need to support ICU nurses in challenging workplace situations. This can be achieved by mentoring them and preparing them towards the grieving process ensuring that the psychosocial aspects of nursing are ingrained in the nursing curriculum and continued into any further branch of specialty nursing such as ICU nursing, Trauma nursing or Paediatric nursing.

\section{Recommendations}

Based on the findings of this study, the following recommendations are suggested with special reference to nursing practice, nursing education, policy development and implementation and further research and are as follows:

\subsection{Nursing Practice}

Nursing as a profession needs to create a support network made up of the multidisciplinary team. Health care settings in South Africa that have an ICU should have a separate grieving area that can facilitate the comfort needs of the family or loved ones of the deceased. Professional assistance and advice should be readily available to all ICU staff requiring debriefing from traumatic ICU events.

\subsection{Nursing Education}

Death education should be emphasized in the South African nursing undergraduate nursing curriculum and continue to be integrated to post-basic ICU training.

\subsection{Policy Development and Implementation}

All nursing staff allocated to work in an ICU environment should undergo an ICU orientation programme on the 
death and dying policies and protocols in an ICU. These policies should comply with the ethical and legal guidelines embedded in the South African constitution, such as information on termination of life support, brain stem death testing and information of organ donation.

\subsection{Further Research}

Further research is recommended to ascertain whether the present South African Diploma in Medical and Surgical Nursing-Critical Care nursing curriculum adequately addresses the issues of death and dying in an ICU. Research into the expectations of the grieving family and the dying patient from the ICU nurse will serve as useful sources for future research reference.

\section{Competing Interests Statement}

The authors declare that there are no competing or potential conflicts of interest.

\section{References}

Alspach, J. G. (2006). Core curriculum for critical care nursing. W.B Saunders Company: Philadelphia.

Beckstrand, R. L., \& Kirchhoff, K. T. (2005). Providing end-of-life care to patients: critical care nurses' perceived obstacles and supportive behaviours. American Journal of Critical Care, 14, 395-403.

Carlson, K. K. (2009). Advanced critical care nursing. Saunders: Canada.

Creswell, J. W. (2007). Qualitative inquiry and research design: Choosing among five approaches. Thousand Oaks: Sage Publications.

de Beer, J., Brysiewicz, P., \& Bhengu, B. R. (2011). Intensive care nursing in South Africa. Southern African Journal of Critical Care, 14, 6-10.

Efstathiou, N., \& Clifford, C. (2011). The critical care nurse's role in End-of-Life care: issues and challenges. Nursing in Critical Care, 16, 116-117. https://doi.org/10.1111/j.1478-5153.2010.00438.x

Fridh, I., Forsberg, A., \& Bergbom, I. (2007). Family presence and environmental factors at time of a patient's death in ICU. Acta Anaesthesiol Scand, 51, 395-401. https://doi.org/10.1111/j.1399-6576.2006.01250.x

Glaser, B. G., \& Strauss, A. L. (2004). Awareness of dying. Aldine: Sociology Press

Hov, R., Hedelin, B., \& Athlin, E. (2005). Being an intensive care nurse related to questions of withholding or withdrawing curative treatment. Journal of Clinical Nursing, (16), 203-211. https://doi.org/10.1111/j.1365-2702.2006.01427.x

Jacobsson, A., Backteman-Erlanson, S., Padyab, M., Egan Sjolander, A., \& Brulin, C. (2016). Burnout and Association with Psychosocial Work Environment among Swedish Firefighters. Global Journal of Health Science, 9(5), 214. https://doi.org/10.5539/gjhs.v9n5p214

Kgosana, A. I., Mamogobo, P. M. Mothiba, T. M., \& Okafor, U. B. (2019). Experiences and Practices of Nurses Caring for Terminally Ill Cancer Patients: A Qualitative Study. Global Journal of Health Science; 11(3), 44-51. https://doi.org/10.5539/gjhs.v11n3p44

Kongsuwan, W., Chaipetch, O., \& Matchim, Y. (2012). Thai Buddhist families 'perspective of a peaceful death in ICUs. Nursing in Critical Care, 17, 156. https://doi.org/10.1111/j.1478-5153.2012.00495.x

Langley, G., \& Schmollgruber, S. (2006). End-of-life care in intensive care units. Southern African Journal of Critical Care, (22), 58-65. https://doi.org/10.1016/j.iccn.2005.03.001

Latour, J. M., Fulbrook, P., \& Albarran, J. W. (2009). EfCCNa survey: European intensive care nurses' attitudes and beliefs towards end-of-life care. Nursing in Critical Care, 14, 110-121. https://doi.org/10.1111/j.1478-5153.2008.00328.x

Liebert, M. (2011). Follow-up study of complicated grief amongst parents eighteen months after a child's death in the paediatric intensive care unit. Journal of Palliative Medicine (online), 14(2), 207-214. https://doi.org/10.1089/jpm.2010.0291

Michell, L. (2010). Crash and burn. Southern African Journal of Critical Care, 26, 34.

Niederriter, J. E. (2009). Student nurses'perception of death and dying (Doctor of Philosophy in Urban Education: Cleveland State University).

Polit, D. F., \& Beck, C. T. (2008). Nursing research: Generating and assessing evidence for nursing practice. Lippincott Williams and Wilkins: Philadelphia. 
Polit, D. F., \& Hungler, B. P. (2004). Nursing research: Principles and methods. Lippincott Williams and Wilkins: Philadelphia.

Press, L. M., Thorn, B., \& Kline, N. (2009). Assessing nurses' attitudes toward death and caring for the dying patient. Journal of Medicine and Biomedical Sciences, 35(6), 955-959. https://doi.org/10.1188/08.ONF.955-959

Prompahakul, B. S. N., Nilmanat, K., \& Kongsuwan, W. (2011). Factors relating to nurses' caring behaviours for dying patients. Nurse Media Journal of Nursing, 1, 15-27.

Urden, L. D., Stacy, K. M., \& Lough, M. E. (2004). Critical care nursing: diagnosis and management. Mosby: St Louis.

Valiee, S., Negarandeh, R., \& Dehghan Nayeri, N. (2012). Exploration of Iranian intensive care nurses' experience of end-of-life care: a qualitative study. Nursing in Critical Care, 17, 315-309. https://doi.org/10.1111/j.1478-5153.2012.00523.x

Wingate, S., \& Wiegand, D. L. (2008). End-of-life care in the critical care unit for patients with heart failure. Critical Care Nurse, 28, 84-95.

\section{Copyrights}

Copyright for this article is retained by the author(s), with first publication rights granted to the journal.

This is an open-access article distributed under the terms and conditions of the Creative Commons Attribution license (http://creativecommons.org/licenses/by/4.0/). 